\title{
Measures of Goodness of a Set of Colour Scanning Filters
}

\author{
Poorvi L. Vora and H. Joel Trussell, \\ Dept. of Electrical and Computer Engineering, \\ North Carolina State University, \\ Raleigh, NC 27695-7911
}

\begin{abstract}
Accurate scanning of a colour image, which is absolutely essential for good colour reproduction, can ensure that all relevant information about the colour stimulus of a signal is obtained. This paper introduces a measure of goodness of a set of colour scanning filters. The q-factor of a single colour scanning filter is shown to be a particular case of the measure. Experimental results are presented to justify the appropriateness of the measure.
\end{abstract}

\section{Introduction}

Most current research in colour systems assumes that the visual frequency spectrum can be adequately represented by samples taken about $10 \mathrm{~nm}$ apart over the range $400-700 \mathrm{~nm}$. Integrals are approximated by summations, and the infinite-dimensional Hilbert space of visible spectra with the usual 2-norm is reduced to an $\mathrm{N}$-dimensional Hilbert space, where $\mathrm{N}$ is the number of samples (in this case $\mathrm{N}=31$ ). A continuous function of wavelength is represented by an $\mathrm{N}$-vector of its sampled values. Hence, visual spectra will be treated as vectors in an $\mathrm{N}$-dimensional Hilbert space in this paper.

The notation in this paper follows that of [1]. Let $\mathbf{S}=\left[\mathbf{s}_{1} \mathbf{s}_{2} \mathbf{s}_{3}\right]$, where $\mathbf{s}_{1}, \mathbf{s}_{2}$ and $\mathbf{s}_{3}$ are N-vectors that represent the colour sensitivity functions of the three types of cones in the eye. Let $\mathbf{A}=\left[\begin{array}{lll}\mathbf{a}_{1} & \mathbf{a}_{2} & \mathbf{a}_{3}\end{array}\right]$ and $\mathbf{P}=\left[\begin{array}{lll}\mathbf{p}_{1} & \mathbf{p}_{2} & \mathbf{p}_{3}\end{array}\right]$ ,where $\mathbf{a}_{i}$ and $\mathbf{p}_{i}(i=1,2,3)$ are $\mathrm{N}$-vectors representing the CIE matching functions and the corresponding CIE primaries, respectively.

For a given reflectance spectrum $\mathbf{f}$ viewed under a uniform radiance source,

$$
\mathbf{S}^{T} \mathbf{f}=\mathbf{S}^{T} \mathbf{P t}
$$

where

$$
\mathbf{t}=\mathbf{A}^{T_{\mathbf{f}}}
$$

The spectra $\mathbf{f}$ and $\mathbf{P t}$ provide identical colour stimuli, and are known as metamers. Thus, the colour stimulus of $\mathbf{f}$ may be reproduced exactly if $\mathbf{t}$, the vector of tristimulus values, is known and the primaries are realisable. Notice that $\mathbf{t}$ is uniquely determined by the projection of $\mathbf{f}$ onto the space spanned by the set of linearly independent vectors, $\left\{\mathbf{a}_{i}\right\}_{i=1}^{3}$ [2], which is also the space spanned by the set of linearly independent vectors, $\left\{\mathbf{s}_{i}\right\}_{i=1}^{3}$. The spectrum $\mathbf{g}$ is a metamer of $\mathbf{f}$ under uniform illumination iff:

$$
\mathbf{A}^{T} \mathbf{f}=\mathbf{A}^{T} \mathbf{g}
$$

i.e. the projection of $\mathbf{g}$ onto the space defined by $\left\{\mathbf{a}_{i}\right\}_{i=1}^{3}$ is identical to the projection of $\mathbf{f}$. 
Suppose the spectrum of the illuminant is represented by $\mathbf{l}$. The reflectance spectrum $\mathbf{f}$ is seen as $\mathbf{L} f$, where $\mathbf{L}$ is a diagonal matrix, such that $\mathbf{L}_{i i}=\mathbf{l}(i)$. For a perfect colour match between the signals $\mathbf{f}$ and $\mathbf{g}$, as viewed under illuminant $\mathbf{l}$,

$$
\mathbf{A}^{T} \mathbf{L f}=\mathbf{A}^{T} \mathbf{L g}
$$

i.e.,

$$
(\mathbf{L A})^{T} \mathbf{f}=(\mathbf{L A})^{T} \mathbf{g}
$$

or

$$
\mathbf{A}_{L}^{T} \mathbf{f}=\mathbf{A}_{L}^{T} \mathbf{g}
$$

and the visual stimulus of a signal is now determined uniquely by its projection onto the space spanned by the set of vectors, $\left\{\mathbf{L a}_{i}\right\}_{i=1}^{3}$. This space is defined as the Human Visual Subspace for the illuminant 1 . The projection is

$$
P_{V} \mathbf{f}=\mathbf{A}_{L}\left(\mathbf{A}_{L}^{T} \mathbf{A}_{L}\right)^{-1} \mathbf{A}_{L}^{T} \mathbf{f}
$$

and is also called the fundamental of $\mathbf{f}$ [3]. The spectrum $\mathbf{g}$ is known as a metamer of $\mathbf{f}$ under illuminant $\mathbf{l}$, and the matrix $\mathbf{A}_{L}$ denotes the matrix product $\mathbf{L A}$.

Colour reproduction begins with correctly determining the projection of a given spectrum onto the HVSS. The set $\left\{\mathbf{L a}_{i}\right\}_{i=1}^{3}$ is not the only basis that may be used for the HVSS. In general, the scanning filters can be any set of vectors that span the HVSS. In this paper, $\left\{\mathbf{a}_{i}\right\}_{i=1}^{3}$ denotes the set of matching functions; $\left\{\mathbf{m}_{i}\right\}_{i=1}^{r}$ denotes any set of $\mathbf{r}$ scanning filters, and $\mathbf{M}$ denotes the matrix of scanning filters:

$$
\mathbf{M}=\left[\mathbf{m}_{1} \mathbf{m}_{2} \ldots \ldots . \mathbf{m}_{r}\right]
$$

The range space of a matrix $\mathbf{X}$ is denoted by $R(\mathbf{X})$. Hence, the HVSS may be denoted by $R(\mathbf{L} \mathbf{A})$, and $R(\mathbf{M})$ denotes the set of linear combinations of the scanning filters. An arbitrary scanning filter is represented by the vector $\mathbf{m}$. A reflectance spectrum whose colour stimulus is to be reproduced is denoted by $\mathbf{f}$.

A number of problems arise in the implementation of a scanning system that obtains the required projection of f. In particular, it is difficult to construct a designed scanning filter exactly, and any errors in filter construction will change the space spanned by the filters, resulting in an error in the measurement of the required projection. This error will lead to an error in the reproduction. Notice that this error will occur even if the measurements are noise-free in all other regards. In an attempt to measure the goodness of a colour filter with respect to such an error, the quality factor or the q-factor of a colour filter was defined by Neugebauer [4]. If $\mathbf{m}$ represents a colour filter and $P_{V}(\mathbf{m})$ its orthogonal projection onto the HVSS, the $q$-factor of $\mathbf{m}$ is defined as:

$$
q(\mathbf{m})=\frac{\left\|P_{V}(\mathbf{m})\right\|^{2}}{\|\mathbf{m}\|^{2}}
$$

where $\|$.$\| is the 2$-norm in $\mathrm{N}$-dimensional vector space. Notice that

$$
0 \leq q(\mathbf{m}) \leq 1
$$

and the closer the value of $q(\mathbf{m})$ to unity, the 'better' the colour scanning filter $\mathbf{m}$. If the value of $q(\mathbf{m})$ is small compared to unity, the filter measures very little of the spectrum that is in the HVSS, and hence is not a good filter for colour scanning. The q-factor seems a reasonable quality measure for filters not in the HVSS, because $\|\mathbf{m}\|^{2}(1-q(\mathbf{m}))$ is the square of the euclidean distance of $\mathbf{m}$ from the HVSS. If any one of a set of three scanning filters, $\left\{\mathbf{m}_{i}\right\}_{i=1}^{3}$ is not in the three-dimensional HVSS (i.e. $q\left(\mathbf{m}_{i}\right) \neq 1$ for some i), then $R(\mathbf{L} \mathbf{A}) \neq R(\mathbf{M})$, and $\left\{\mathbf{m}_{i}\right\}_{i=1}^{3}$ is inaccurate for colour sensing. A major disadvantage of the q-factor is that it is designed to be used with only a single filter.

A measure that extends the idea of the q-factor to judge the effectiveness of a set of colour scanning filters would be very useful. Notice that the q-factor can be used to measure the goodness of the filter as a member of a set of three scanning filters in a limited sense. For example, it does not indicate if the filters are linearly independent. In most 
existing scanning systems, only three scanning filters are used. Three linearly independent scanning filters can span the three-dimensional HVSS iff all three have unit q-factors. Hence the q-factor indicates a perfect set of filters if

$$
q\left(\mathbf{m}_{i}\right)=1 \text { for every } i
$$

and the $\mathbf{m}_{i}$ are linearly independent vectors. The $\mathrm{q}$-factor does not assist in differentiating among imperfect sets of vectors, and may not be used by itself to indicate a 'better' imperfect set of filters. Another disadvantage of the $\mathrm{q}$-factor is that it may at best be used to judge a set of three filters.

There are at least two reasons why more than three filters may be used to improve the quality of the colour reproduction. Firstly, in many cases, three parameters are not enough to define sufficiently the visual stimulus of an $\mathrm{N}$-dimensional signal for colour correction. Typically, such a situation arises when the colour reproduction is to be viewed under two different illuminants. In such a case, six parameters (representing the projections of the signal onto the two different Human Visual Subspaces defined by the two different illuminants) are required to accurately represent the signal [1]. Secondly, the constraint of constructabilty on the filters might imply that no set of three constructable filters can span the HVSS though a set of four filters could be constructed so that the required projection is obtained. When more than three parameters, (four scanning filters, for example) are necessary, the q-factor is not an effective measure of the goodness of even each single filter as part of the set of more than three filters. For example, suppose $\left\{\mathbf{m}_{i}\right\}_{i=1}^{4}$ is a set of scanning filters. It is possible that the HVSS is contained in the span of the set of four filters, i.e.:

$$
R(\mathbf{M}) \supseteq R(\mathbf{A})
$$

but,

$$
q\left(\mathbf{m}_{i}\right)<1 \quad i=1,2,3,4
$$

Such a set could provide perfect colour scanning, though the individual q-factors would not indicate this. An example of such a set is presented in Section 4.

The performance of a set of filters can be judged by the reproduction quality of a set of signals. Usually this set of signals is chosen so as to represent the ensemble of signals which will be scanned. The average error in the reproduction is often used as an indicator of the goodness of the set of filters. The q-factor of a colour scanning filter has the disadvantage of not being a good indicator of the perceptual error in colour reproduction.

As just discussed, the q-factor has three major disadvantages. Firstly, it measures a single filter independently of other filters in the scanning set. A measure that extends the idea of the q-factor to judge the effectiveness of a set of colour scanning filters would be very useful. Secondly, it can be used to judge the merit of a single filter as part of a set of three filters only in the limited sense of determining if the set spans the HVSS. Thirdly, it is not useful in judging the merit of a single filter as part of a set of more than three filters. It is possible to develop a measure that overcomes these disadvantages. Section 3 presents such a measure. The relationship of the proposed measure with the $\mathrm{q}$-factor is discussed. Section 2 deals with the requirements of an effective measure.

\section{Requirements of an Effective Measure}

If a set of scanning filters is linearly independent, the measurements obtained (the values of $\mathbf{m}_{i}^{T} \mathbf{f}$ ) will be nonredundant and can be used to obtain the projection of the measured spectrum onto the space spanned by the scanning filters. If these filters are not linearly independent, all measurements are not necessary to find the projection onto the space spanned by the filters. In this case, the extra measurements may help eliminate noise in the scanning process. In either case, a measure of goodness of the filter set is related to the space spanned by the scanning filters, and the relation of this space with the HVSS. This measure should not be related to the noise performance of the filters, as it should measure performance of the filters with respect to an error that occurs independent of any additional measurement noise.

An effective measure of goodness of a set of scanning filters should satisfy the following conditions. Firstly, the measure should depend only on the space spanned by the scanning filters, and not on particular, individual filters. 
For perfect scanning, the HVSS should be contained in the space spanned by the scanning filters, as indicated in equation (11). Hence, a second requirement is that the measure should indicate a perfect set of scanning filters. When the scanning is not perfect, the measure should distinguish among filter sets according to the goodness of the approximation to projections in the HVSS. Thirdly, the measure should be generalizable to an arbitrary number of filters and an arbitrary reproduction space.

Let $\left\{\mathbf{v}_{i}\right\}_{i=1}^{s}$ denote a set of vectors that define the space to be spanned, then the space to be spanned is denoted by $R(\mathbf{V})$, where,

$$
\mathbf{V}=\left[\mathbf{v}_{1} \mathbf{v}_{2} \ldots \ldots . \mathbf{v}_{\mathbf{s}}\right]
$$

It is necessary to note here that $R(\mathbf{V})$ need not be three-dimensional; for example, effective colour correction requires the projection onto a space of dimension greater than three [1]. The dimensions of $R(\mathbf{V})$ and $R(\mathbf{M})$ may not be equal; a large set of filters $\left\{\mathbf{m}_{i}\right\}_{i=1}^{r}$ may be used to ensure the spanning of $R(\mathbf{V})$.

A measure that immediately comes to mind is the dimension of the intersection of $R(\mathbf{M})$ and $R(\mathbf{V})$. On closer examination this measure is found to be too coarse. It takes on only integer values, and does not distinguish well enough between 'good' and 'not-so-good' sets of scanning filters. For example, consider the space spanned by the Nvectors $\mathbf{v}_{1}=[1,0,0,0, \ldots . .0]^{T}, \mathbf{v}_{2}=[0,1,0,0, \ldots \ldots .0]^{T}$ and $\mathbf{v}_{3}=[0,0,1,0, \ldots \ldots .0]^{T}$ as the space to be spanned. Let the N-vectors $\mathbf{m}_{1}=[1,0,0,0, \ldots \ldots .0]^{T}, \mathbf{m}_{2}=[0,0,1,0, \ldots \ldots .0]^{T}$ and $\mathbf{m}_{3}=[0,0,0,1, \ldots \ldots .0]^{T}$ be the scanning filters. The dimension of the intersection, $R(\mathbf{V}) \cap R(\mathbf{M})$ is two. Now suppose $\mathbf{m}_{1}^{\prime}=[1,0,0,0, \ldots \ldots .0]^{T}, \mathbf{m}_{2}^{\prime}=[0,0.95,1,0, \ldots \ldots . .0]^{T}$ and $\mathbf{m}_{3}^{\prime}=[0,0,0,1, \ldots . .0]^{T}$ is another set of scanning filters. The dimension of the intersection is also two, but the second set will provide much more information of the required projection. A measure is needed that will distinguish between such sets of filters.

The problem of finding a measure of the goodness of a set of scanning filters is directly related to the error associated with such a set, in the absence of any additional noise. The error is defined as the difference between the required signal and the signal obtained with the scanning filter set. This error depends on the particular reflectance vector, f, which is being measured. As the human eye is sensitive only to errors in the Human Visual Space, the error may be defined as the difference between the actual and the reconstructed fundamentals. It is common to consider an average error over some well-defined set of reflectance spectra, $\left\{\mathbf{f}_{k}\right\}$. Among the many error measures that may be used to judge the performance of a filter set are the mean square error, the mean square tristimulus error and the mean square error in a uniform colour space such as the $C I E L^{*} a^{*} b^{*}$ space. Two of these errors are studied in this paper, but before an error expression can be obtained, some notation needs to be established.

Let an orthonormal basis for $R(\mathbf{V})$ be defined:

$$
\mathbf{N}=\left[\begin{array}{llll}
\mathbf{n}_{1} & \mathbf{n}_{2} & \ldots . . . \mathbf{n}_{\alpha}
\end{array}\right]
$$

such that:

$$
R(\mathbf{N})=R(\mathbf{V})
$$

and:

$$
\mathbf{N}^{T} \mathbf{N}=\mathbf{I}
$$

which may be obtained by the Gram-Schmidt orthogonalisation procedure [6]. The number of orthonormal vectors, $\alpha$, is the rank of $\left\{\mathbf{v}_{i}\right\}_{i=1}^{s}$ and $\alpha$ equals $s$ iff $\left\{\mathbf{v}_{i}\right\}_{i=1}^{s}$ is a linearly independent set. Similarly, define an orthonormal basis for $R(\mathbf{M})$ :

$$
\mathbf{O}=\left[\begin{array}{llll}
\mathbf{o}_{1} & \mathbf{o}_{2} & \ldots \ldots . \mathbf{o}_{\beta}
\end{array}\right]
$$

such that:

$$
R(\mathbf{O})=R(\mathbf{M})
$$

and:

$$
\mathbf{O}^{T} \mathbf{O}=\mathbf{I}
$$

Again, notice that $\beta$ is the rank of $\left\{\mathbf{m}_{i}\right\}_{i=1}^{r}$ and $\beta$ equals $r$ iff $\left\{\mathbf{m}_{i}\right\}_{i=1}^{r}$ is a linearly independent set. The orthonormal bases $\mathbf{N}$ and $\mathbf{O}$ need not represent realisable filters. Let $P_{M}($.$) represent the orthogonal projection operator onto$ 
$R(\mathbf{M})$ in N-space. Similarly, let $P_{V}($.$) represent the orthogonal projection operator onto R(\mathbf{V})$ in N-space. Define:

$$
P_{V}(\mathbf{O})=\left[P_{V}\left(\mathbf{o}_{1}\right) P_{V}\left(\mathbf{o}_{2}\right) \ldots \ldots P_{V}\left(\mathbf{o}_{\beta}\right)\right]
$$

and likewise:

$$
P_{M}(\mathbf{N})=\left[P_{M}\left(\mathbf{n}_{1}\right) P_{M}\left(\mathbf{n}_{2}\right) \ldots \ldots . P_{M}\left(\mathbf{n}_{\alpha}\right)\right]
$$

The projection of a visual spectrum $\mathbf{f}$ onto $R(\mathbf{V})$ is the information required for accurate colour reproduction as defined by the designers of the reproduction system. This required projection of a visual spectrum $\mathbf{f}$ onto $R(\mathbf{V})$ is

$$
P_{V}(\mathbf{f})=\mathbf{N N}^{T} \mathbf{f}
$$

In the special case where $R(\mathbf{V})$ is the HVSS, $P_{V}(\mathbf{f})$ is the fundamental of the spectrum $\mathbf{f}$ [3]. The projection obtained using the scanning filters $\left\{\mathbf{m}_{i}\right\}_{i=1}^{s}$ is the projection onto $R(\mathbf{M})$ :

$$
P_{M}(\mathbf{f})=\mathbf{O O}^{T} \mathbf{f}
$$

The projection of this onto the HVSS is

$$
P_{V} P_{M}(\mathbf{f})=\mathbf{N N}^{T} \mathbf{O O}^{T} \mathbf{f}
$$

In the special case where $R(\mathbf{V})$ is the HVSS, $P_{V} P_{M}(\mathbf{f})$ is the estimated fundamental of the spectrum $\mathbf{f}$, and is the relevant information about $f$ that can be obtained from the scanned data. Expressions (22) and (25) give the required projection and the obtained projection, respectively. Clearly, the most important criterion of a measure of goodness is that it must provide a good indication of the error in the reproduction. The next section deals with the mean square error measure for colour reproduction, and a measure of goodness that is directly related to this error measure.

The requirements of a valid measure of goodness may be summarized as follows. Firstly, the measure must be indicative of a perfect filter set, and of colour reproduction errors when the filter set is imperfect. Secondly, the measure must be independent of the particular filters used and dependent only on the space spanned by the scanning filters. Thirdly, it should be generalizable to an arbitrary dimension of $R(\mathbf{V})$ and to an arbitrary number of filters.

\section{An Error Measure and a Related Measure of Goodness}

Two major error measures are often used in the evaluation of colour reproductions, mean square error and mean $L^{*} a^{*} b^{*}$ error. In either case, the mean error indicates an average over a particular data set, and is hence dependent on the data set. The problems of correlating mean square error with perceptual error are well known. The mean square error is addressed because it is easy to manipulate and because its analysis provides valuable insight into the problem of reproduction errors due to filter construction errors. The mean $\Delta_{L^{*} a^{*} b^{*}}$ error measure is far more difficult to analyse and manipulate, but is a valid perceptual error measure. While there are cases where colour estimates may have low mean square errors and high $\Delta_{L^{*} a^{*} b^{*}}$ errors and vice versa, the average of the errors over a data set are generally in qualitative agreement, as demonstrated in Section 5.

\subsection{Mean Square Error}

The error between the two fundamentals $P_{V}(\mathbf{f})$ and $P_{V} P_{M}(\mathbf{f})$ is the difference between expressions (23) and (25):

$$
P_{V} \mathbf{e}=P_{V}(\mathbf{f})-P_{V} P_{M}(\mathbf{f})=\mathbf{N N}^{T} \mathbf{f}-\mathbf{N N}^{T} \mathbf{O O}^{T} \mathbf{f}
$$

It can be shown that the mean square value of the components of $P_{V}(\mathbf{e})$ is:

$$
E\left[\left\|P_{V}(\mathbf{e})\right\|^{2}\right]=\operatorname{Trace}\left(\mathbf{N}^{T}\left(\mathbf{I}-\mathbf{O O}^{T}\right) \mathbf{R}\left(\mathbf{I}-\mathbf{O O}^{T}\right) \mathbf{N}\right)
$$

where $E[$.$] is the expectation operator over the particular ensemble and \mathbf{R}$ is $E\left[\mathbf{f f}^{T}\right]$. This expression may not be simplified further unless assumptions are made about the nature of $\mathbf{R}$. For a given sample set, however, this error is easily computed and will be useful for many who now use sample sets anyway. 


\subsection{Error for Independent, Identically Distributed Components}

In the particular case when $\mathbf{R}$ is a scalar multiple of the identity matrix, i.e. when the spectrum $\mathbf{f}$ can be expressed as a sequence of independent, identically distributed random variables, the error expression is considerably simplified. It is then:

$$
E\left[\left\|P_{V}(\mathbf{e})\right\|^{2}\right]=\sigma^{2} \operatorname{Trace}\left(\mathbf{N}^{T}\left(\mathbf{I}-\mathbf{O O}^{T}\right)\left(\mathbf{I}-\mathbf{O O}^{T}\right) \mathbf{N}\right)
$$

where $\sigma^{2}$ is the variance of a single component of $\mathbf{f}$. This simplifies to

$$
E\left[\left\|P_{V}(\mathbf{e})\right\|^{2}\right]=\sigma^{2}\left(\sum_{i=1}^{\alpha}\left(1-\lambda_{i}^{2}\left(\mathbf{O}^{T} \mathbf{N}\right)\right)\right)
$$

where $\lambda_{i}\left(\mathbf{O}^{T} \mathbf{N}\right)$ denotes the $i^{\text {th }}$ singular value of $\left(\mathbf{O}^{T} \mathbf{N}\right)$. Perfect reproduction is possible when:

$$
\alpha=\sum_{i=1}^{\alpha} \lambda_{i}^{2}\left(\mathbf{O}^{T} \mathbf{N}\right)
$$

or,

$$
\lambda_{i}\left(\mathbf{O}^{T} \mathbf{N}\right)=1 \quad i=1, \ldots \alpha
$$

which happens when

$$
R(\mathbf{M}) \supseteq R(\mathbf{V})
$$

It may be noted that the conditions for perfect reproduction are independent of the variance of the components of $f$. The term $\alpha$ in expression (28) is the dimension of the space to be spanned, and is assumed invariate. The summation may be used as a measure of goodness of the filters, as a high value of the summation indicates a low error. It can be shown that,

$$
\lambda_{i}^{2}\left(\mathbf{O}^{T} \mathbf{N}\right) \leq 1 \quad i=1, \ldots \alpha
$$

and a normalised measure of goodness of the filter set is:

$$
\nu(\mathbf{V}, \mathbf{M})=\frac{\sum_{i=1}^{\alpha} \lambda_{i}^{2}\left(\mathbf{O}^{T} \mathbf{N}\right)}{\alpha}
$$

Notice that

$$
\nu(\mathbf{V}, \mathbf{M})=1 \text { iff } R(\mathbf{M}) \supseteq R(\mathbf{V})
$$

Hence the measure is unity if and only if the set of scanning filters is perfect.

\subsection{Relationship with Q-factor}

It can be shown that the measure of goodness defined in expression (34) is related to Neugebauer's q-factor measure. Specifically, this measure of goodness is the sum of the q-factors of the $\mathbf{o}_{\boldsymbol{i}}$ divided by the dimension of $R(\mathbf{V})$. Hence,

$$
\sum_{i=1}^{\alpha} \lambda_{i}^{2}\left(\mathbf{N}^{T} \mathbf{O}\right)=\sum_{i=1}^{\beta} q \text { factor }\left(\mathbf{o}_{i}\right)
$$

Thus the sum of $\mathbf{q}$-factors of the filters represented by $\mathbf{O}$, an orthonormal basis for the space spanned by the scanning filters, is a valid measure of goodness for the set of filters, when the signal $f$ is from an ensemble of independent identically distributed random variables. The sum may be normalised so that a maximum value of unity indicates perfect reproduction. This normalised form is:

$$
\nu(\mathbf{V}, \mathbf{M})=\frac{\sum_{i=1}^{\beta} q \text { factor }\left(\mathbf{o}_{i}\right)}{\alpha}
$$


Here the term q-factor is used in a general sense to mean the norm of the projection of the normalised vectors $\mathbf{o}_{i}$ onto the $\alpha$-dimensional $R(\mathbf{V})$. Neugebauer's original definition of the q-factor implies the specific case where this space is the three-dimensional HVSS.

A necessary condition for the measure to be independent of the particular set of filters used to span the respective spaces is that the filters $\mathbf{O}$ should be orthonormal. So, for example, $\sum_{i=1}^{r} q$ factor $\left(\mathbf{m}_{i}\right)$ cannot be used as a measure instead of $\sum_{i=1}^{\beta} q$ factor $\left(\mathrm{o}_{i}\right)$. To see that the normalised sum of the q-factors of the scanning filters themselves is not a valid measure consider the following example:

Let $\mathbf{v}_{1}=[1,0,0]^{T}$, and $\mathbf{v}_{2}=[0,1,0]^{T}$ be the vectors representing a simple three-dimensional HVSS. Let $\mathbf{m}_{1}=[1,0,0]^{T}$, and $\mathbf{m}_{2}=[0, \operatorname{Cos}(\pi / 3), \operatorname{Sin}(\pi / 3)]^{T}$ be vectors representing the scanning filters. The sum of the q-factors is 1.25 . Now, suppose another set of scanning filters is chosen, that spans the same space as the first set. Suppose the set is $\mathbf{m}_{1}=[1,0,0]$, and $\mathbf{m}_{2}=\left[\frac{2}{5}, \frac{\operatorname{Cos}(\pi / 3)}{5}, \frac{\operatorname{Sin}(\pi / 3)}{5}\right]$. Notice that this set of scanning filters is not orthogonal. The sum of $\mathrm{q}-$ factors for this set is 1.85 . Hence,

$$
\phi(\mathbf{V}, \mathbf{M})=\sum_{i=1}^{2} q \text { factor }\left(\mathbf{m}_{i}\right)
$$

is seen to be dependent on the particular filters used, and is not just a function of the space spanned by the filters, unless the orthogonality condition is imposed on the filters. Hence it is not a valid measure of goodness. Note that if the filters $\mathbf{m}_{i}$ are narrowband with little overlap, they are "close" to orthogonal. In this case $\phi(\mathbf{V}, \mathbf{M})$ may be a good approximation to $\nu(\mathbf{V}, \mathbf{M})$. The measure $\nu(\mathbf{V}, \mathbf{M})$ avoids this problem entirely.

If each scanning filter is to be evaluated by itself, and not as part of a larger set of filters, the proposed measure can be used to evaluate the set consisting of a single scanning filter. In this case, the proposed measure is exactly the same as the q-factor of Neugebauer, and Neugebauer's definition of the q-factor may be seen as a specific instance of the measure defined in (34).

\section{A Perfect Filter Set}

In Section 1, it was mentioned that it is possible that a four-filter set can ensure perfect colour scanning. An example is presented here of an imperfect three-filter set with fairly high individual q-factors. It is demonstrated that the addition of a fourth filter to the set makes the set perfect, though the q-factor of the fourth filter is very low.

Consider the set of three all-positive filters shown in Figs. 1-3. This set of filters has a measure, $\nu(\mathbf{A}, \mathbf{M})$, of 0.953 . The filters have q-factors of $0.873,0.891$ and 0.939 respectively. Clearly, this set does not span the HVSS, and is not a perfect set of scanning filters. The mean square error between the fundamental and the estimated fundamental for a 64 signal subset of the Munsell chip set is 0.188 , and the average $\Delta L^{*} a^{*} b^{*}$ error for the same set is 3.4. The addition of a fourth filter to the set, shown in Fig. 4, increases the measure to 1.0, and the resulting four-filter set spans the HVSS. The q-factor of the fourth filter is 0.246 . This is an example of a case where the q-factor of a filter is not indicative of its appropriateness as a scanning filter.

\section{Experimental Results}

Several ensembles were used to study the appropriateness of the proposed measure, $\nu(\mathbf{V}, \mathbf{M})$. The results presented below used a 64 sample set of Munsell chips. Filter Set 1 is the set of Kodak Wratten gelatin filter Nos. 52, 49, 72B. Filter Set 2 is the above set with a fourth filter added, the Kodak Wratten gelatin filter No. 57. Filter Set 3 consists of a five filters, the four in Set 2 and Kodak Wratten gelatin filter No. 30. Filter Set 4 is the set of filters shown in Figs. 1-3. The mean-square error and the $\Delta L^{*} a^{*} b^{*}$ error are tabulated with the measure $\nu(\mathbf{A}, \mathbf{M})$. 


\begin{tabular}{||c|c|c|c||}
\hline Filter Set & Measure & Mean Square Error & $\Delta E_{L^{*} a^{*} b^{*}}$ \\
\hline 1 & 0.858 & 0.188 & 16.41 \\
\hline 2 & 0.913 & 0.152 & 15.73 \\
\hline 3 & 0.943 & 0.004 & 2.43 \\
\hline 4 & 0.953 & 0.015 & 3.40 \\
\hline
\end{tabular}

Notice that the mean-square error and the $\Delta L^{*} a^{*} b^{*}$ error behave similarly. A reasonable conclusion is that the average mean square error is a good indicator of the average perceptual error. The measure is not always a good indicator of the mean-square error, as the data set used is not composed of signals with independent, identically distributed (iid) components. This is also a problem with the q-factor, which gives no indication of perceptual error.

\section{Conclusions}

The proposed measure is a valid measure of goodness of a filter set with respect to the mean square error between the fundamental and its estimate, when the signal components are iid. If the signal components are not iid, the proposed measure is at least as good as the q-factor, and does eliminate some of the disadvantages of the q-factor. The mean square error is usually a good indicator of the average $\Delta L^{*} a^{*} b^{*}$ error.

\section{References}

[1] TRUSSELL, H. J.,Applications of Set Theoretic Methods to Color Systems, Color Research and Application, Vol. 16, No. 1, pp 31-41, Feb 1991.

[2] SHAPIRO, W. A., Generalisation of Tristimulus Coordinates, Journal of the Optical Society of America, Vol. 56, No 6, pp 795-802, June 1966.

[3] COHEN, J. B., and KAPPAUF, W. E., Metameric Color Stimuli, Fundamental metamers and Wyszecki's Metameric Blacks, American Journal of Psychology, Vol. 95, pp 537-564, 1982.

[4] NEUGEBAUER, H. E. J., Quality Factor for Filters Whose Spectral Transmittances are Different from Color Mixture Curves, and its Application to Color Photography, Journal of the Optical Society of America, Vol. 46, No. 10, pp 821-824, Oct 1956.

[5] PRATT, W. K., Digital Image Processing, Wiley, 1991.

[6] HORN, R. A., and JOHNSON, C. R., Matrix Analysis, Cambridge University Press, 1985. 


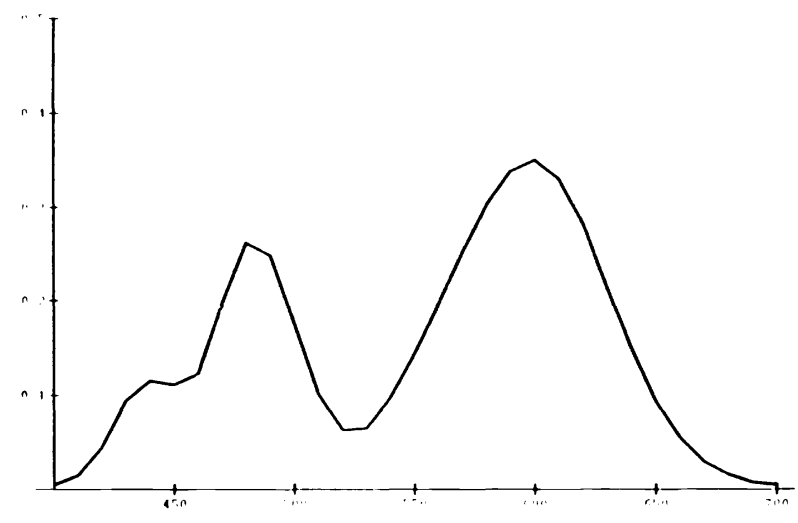

Figure 1

First scanning filter, q-factor $=0.873$

(transmission vs. wavelength)

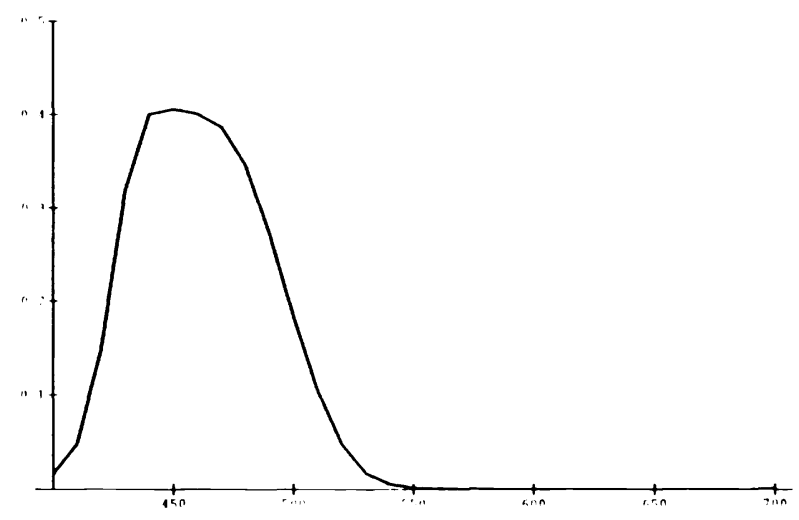

Figure 3

Third scanning filter, $\mathrm{q}$-factor $=0.939$ (transmission vs. wavelength)

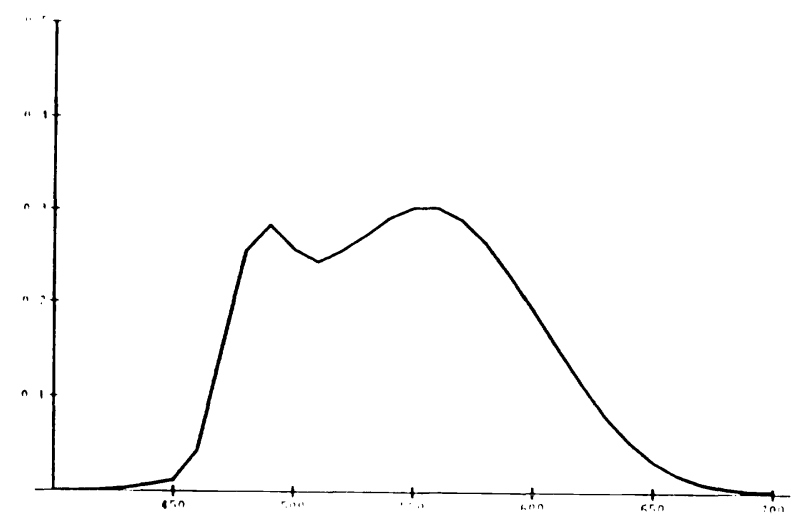

Figure 2

Second scanning filter, $\mathrm{q}$-factor $=0.891$ (transmission vs. wavelength)

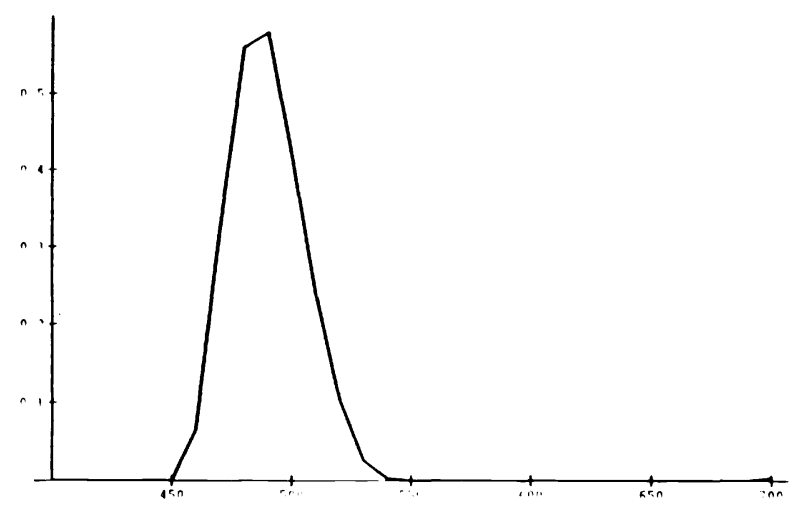

Figure 4

Fourth scanning filter, $\mathrm{q}$-factor $=0.246$ (transmission vs. wavelength) 